\title{
ALL TYPES IMPLIES TORSION
}

\author{
WALTER PARRY
}

(Communicated by Warren J. Wong)

\begin{abstract}
We prove the following theorem. Given a positive integer $n$ and a subset $A$ of $\mathbf{Z}^{n}$ with the following properties: (1) for every $\left(a_{1}, \ldots, a_{n}\right)$ in $A$ the inequality $\sum_{i=1}^{n}\left|a_{i}\right| \geq 2$ holds, and (2) for every $\left(x_{1}, \ldots, x_{n}\right)$ in $\mathbf{R}^{n}$ there exists an $\left(a_{1}, \ldots, a_{n}\right)$ in $A$ with $a_{i} x_{i} \geq 0$ for $i=1, \ldots, n$, there exists a subset $A_{0}$ of $A$ such that $\mathbf{Z}^{n}$ modulo the subgroup generated by $A_{0}$ contains a nontrivial torsion element.
\end{abstract}

\section{INTRODUCTION}

In [1] Gordon and Luecke prove that if two knots in $S^{3}$ have homeomorphic complements, then they are equivalent. The purpose of this paper is to solve a problem that arose in the course of their work.

The statement of the problem requires some definitions. Let $n$ be a positive integer. An n-type is an element of $\{ \pm 1\}^{n}$, namely, an $n$-tuple of 1's and -1 's. An element $\left(a_{1}, \ldots, a_{n}\right)$ in $\mathbf{Z}^{n}$ represents an $n$-type $\tau$ if

(i) $\sum_{i=1}^{n}\left|a_{i}\right| \geq 2$ and

(ii) if $\tau=\left(e_{1}, \ldots, e_{n}\right)$, then there exists $e= \pm 1$ such that $\operatorname{sgn}\left(a_{i}\right)=e e_{i}$ for all $i$ such that $a_{i} \neq 0$.

A subset $A$ of $\mathbf{Z}^{n}$ represents all $n$-types if for every $n$-type $\tau$ there exists an element in $A$ which represents $\tau$. At the beginning of $\S 3$ of [1] Gordon and Luecke conjecture that if a subset $A$ of $\mathbf{Z}^{n}$ represents all $n$-types, then $A$ contains a subset $A_{0}$ such that $\mathrm{Z}^{n}$ modulo the subgroup generated by $A_{0}$ contains a nontrivial torsion element (all types implies torsion). The purpose of this paper is to prove this conjecture. It will be convenient to reformulate the statement of the conjecture and formally state the result as follows. By replacing the above $A$ by $A \cup(-A)$, it is easy to see that the two statements are equivalent.

Theorem. Suppose a positive integer $n$ and a subset $A$ of $\mathbf{Z}^{n}$ with the following properties: for every $\left(a_{1}, \ldots, a_{n}\right)$ in $A$ the inequality $\sum_{i=1}^{n}\left|a_{i}\right| \geq 2$ holds and for every $\left(x_{1}, \ldots, x_{n}\right)$ in $\mathbf{R}^{n}$ there exists an $\left(a_{1}, \ldots, a_{n}\right)$ in $A$ with $a_{i} x_{i} \geq 0$

Received by the editors July 21, 1989 and, in revised form, February 26, 1990.

1980 Mathematics Subject Classification (1985 Revision). Primary 20K15. 
for $i=1, \ldots, n$. Then there exists a subset $A_{0}$ of $A$ such that $\mathbf{Z}^{n}$ modulo the subgroup generated by $A_{0}$ contains a nontrivial torsion element.

Remark. The introduction of $\mathbf{R}^{n}$ is not essential. It can be used to give a geometric interpretation of the main condition on $A$ as follows. Two-dimensional Euclidean space $\mathbf{R}^{2}$ can be subdivided into four quadrants in a familiar way. Let a closed standard sector of $\mathbf{R}^{n}$ be the straightforward generalization of a closed quadrant in $\mathbf{R}^{2}$. Then the main condition on $A$ is that $A$ contains a point in every closed standard sector of $\mathbf{R}^{n}$.

This result might be viewed as simplifying [1] because it eliminates the need for $\S 3$. Also, it will hopefully be applicable to related problems. I wish to thank Cameron Gordon for his kindness in the course of this work.

\section{Proof of THE THEOREM}

The proof will proceed by contradiction. Suppose that the theorem is false, namely, that $A$ as in the theorem is given and the following holds.

If $A_{0}$ is a subset of $A$, then $\mathbf{Z}^{n}$ modulo the subgroup generated by $A_{0}$ is torsionfree.

This will lead to a contradiction.

First choose elements $b_{1}, \ldots, b_{m}$ in $A$ that form a maximal linearly independent subset of $A$. In particular, the subspace of $\mathbf{R}^{n}$ spanned by $A$ has dimension $m$. By (1) there exist elements $b_{m+1}, \ldots, b_{n}$ in $\mathbf{Z}^{n}$ such that $b_{1}, \ldots, b_{n}$ form a basis of $\mathbf{Z}^{n}$. Define, as usual, the standard inner product from $\mathbf{R}^{n} \times \mathbf{R}^{n}$ to $\mathbf{R}$ so that if given $x=\left(x_{1}, \ldots, x_{n}\right)$ and $y=\left(y_{1}, \ldots, y_{n}\right)$ in $\mathbf{R}^{n}$, then $x \cdot y=\sum_{i=1}^{n} x_{i} y_{i}$. Let $b_{1}^{*}, \ldots, b_{n}^{*}$ form the basis dual to the basis formed by $b_{1}, \ldots, b_{n}$ for the standard inner product. Then $b_{1}^{*}, \ldots, b_{n}^{*}$ also form a basis of $\mathbf{Z}^{n}$. Thus it is possible to choose $c_{j}=0$ or 1 for $j=1, \ldots, n$ such that

$$
\sum_{j=1}^{n} c_{j} b_{j}^{*} \equiv(1,1,1, \ldots, 1) \quad \bmod 2 .
$$

Reorder $b_{1}, \ldots, b_{m}$ and $b_{1}^{*}, \ldots, b_{m}^{*}$ if necessary so that $c_{j}=1$ for $j=$ $1, \ldots, l$ and $c_{j}=0$ for $j=l+1, \ldots, m$ for some integer $l$ with $0 \leq l \leq m$.

It will next be shown that proving the theorem reduces to proving that the assumption (1) leads to the following.

It is possible to choose $e_{j}= \pm 1$ for $j=1, \ldots, l$ and $e_{j}=1$ for $j=l+1, \ldots, n$ such that for $x=\sum_{j=1}^{n} e_{j} c_{j} b_{j}^{*}$ and every $a$ in $A, a \cdot x \in\{-1,0,1\}$.

For this reduction, suppose that (2) is true and $x=\left(x_{1}, \ldots, x_{n}\right)$. Then $x_{i} \equiv 1$ $\bmod 2$ for $i=1, \ldots, n$, and hence $x_{i} \neq 0$ for $i=1, \ldots, n$. Choose $a=\left(a_{1}, \ldots, a_{n}\right)$ in $A$ with $a_{i} x_{i} \geq 0$ for $i=1, \ldots, n$. The fact that $a \cdot x \in\{-1,0,1\}$ immediately gives a contradiction. Thus proving the theorem reduces to deriving (2) from (1). 
Remark. In order for (2) to give a contradiction, it is crucial that $x_{i} \neq 0$ for $i=1, \ldots, n$. These inequalities are implied by the congruences $x_{i} \equiv 1 \bmod 2$ for $i=1, \ldots, n$. For these congruences to hold it suffices for every $e_{j}$ to be an odd integer. However, since $a \cdot x \in\{-1,0,1\}$ for every $a$ in $A$, $b_{j} \cdot x \in\{-1,0,1\}$ for $j=1, \ldots, l$. This implies that $e_{j} \in\{-1,0,1\}$ for $i=1, \ldots, l$. On the other hand, $e_{l+1}, \ldots, e_{n}$ can be taken to be any odd integers. In (2) they are taken to be 1 for simplicity.

To derive (2) from (1), first note that the case $l=0$ is trivial, so suppose that $l>0$. Further suppose that the elements of $A$ are $a_{1}, \ldots, a_{k}$ (it may be assumed that $A$ is finite). Define a $k \times l$ matrix $M$ of integers as follows. The $i$ th row $M_{i}$ of $M$ gives the first $l$ coefficients of $a_{i}$ with respect to the basis $b_{1}, \ldots, b_{n}$ of $\mathbf{Z}^{n}$. In other words, if $a_{i}=\sum_{j=1}^{n} m_{i j} b_{j}$ for integers $m_{i j}$, then $M_{i}=\left(m_{i 1}, \ldots, m_{i l}\right)$. Let $N$ be an $h \times h$ matrix gotten from $M$ by deleting some (possibly none) rows and columns for some positive integer $h$. Let $A_{0}$ be the subset of $A$ consisting of the elements $a_{i}$ for which the $i$ th row is not deleted, the elements $b_{j}$ for which the $j$ th column is deleted and $b_{l+1}, \ldots, b_{m}$. Then $\mathbf{Z}^{n}$ modulo the subgroup generated by $A_{0}$ is isomorphic with $\mathbf{Z}^{h}$ modulo the subgroup generated by the rows of $N$. Since this quotient group is torsionfree, the invariant factor theorem implies that $\operatorname{det}(N) \in\{-1,0,1\}$. In particular, the entries of $M$ are all -1 or 0 or 1 . Consequently, it is easy to see that deriving (2) from (1) in turn reduces to proving the following.

Let $M$ be a $k \times l$ matrix of integers with rank $l$ such that if $N$ is a square matrix gotten from $M$ by deleting some (possibly none) rows and columns, then $\operatorname{det}(N) \in\{-1,0,1\}$. Then there exists $e=\left(e_{1}, \ldots, e_{l}\right)$ with $e_{j}= \pm 1$ for $j=1, \ldots, l$ such that $M_{i} \cdot e \in\{-1,0,1\}$ for $i=1, \ldots, k$, where $M_{i}$ is the $i$ th row of $M$.

Here are two observations concerning (3). The condition $\operatorname{det}(N) \in\{-1,0,1\}$ in (3) easily implies that

the rank of every submatrix of $M$ equals the rank of its reduction modulo 2 .

Also, the columns of $M$ are linearly independent, which easily implies that every matrix gotten from $M$ by simply deleting columns also satisfies the assumptions of (3).

It remains to prove (3). As a prelude to this, note that although in the present case the rank of $M$ is $l$, the case in which such a matrix $M$ has lower rank can be reduced to the present case simply by enlarging $M$ by adjoining elementary row vectors. Here, as usual, an elementary row vector is a row vector whose entries are 0 except for one 1 . Define elementary column vectors analogously. The proof of (3) will now proceed by induction on $r$, the number of nonelementary columns in $M$.

If $r=0$, then the columns of $M$ are all elementary and they are distinct because the rank of $M$ is $l$. Thus in this case it is possible to choose $e_{j}=1$ for 
$j=1, \ldots, l$. So, assume that $r>0$ and that $(3)$ is true for smaller values of $r$. Note that there exists a row $M_{i}$ in $M$ with $M_{i} \cdot(1,1,1, \ldots, 1) \equiv 1 \bmod 2$ for otherwise the reduction of $M$ modulo 2 has rank less than $l$, contrary to (4). Without loss of generality, assume that $M_{1} \cdot(1,1,1, \ldots, 1) \equiv 1 \bmod 2$.

The proof of (3) will be completed with another induction argument after line (7). The discussion from here to (7) is a preparation for that. Assume the following.

The dimension of the span of $M_{2}, \ldots, M_{k}$ is less than $l$, namely, $l-1$.

Reorder $M_{2}, \ldots, M_{k}$ if necessary so that $M_{1}, \ldots, M_{l}$ are linearly independent, and let $N$ be the $l \times l$ matrix which they form. Consider the first row cofactors of $N$. They are all -1 or 0 or 1 . They form a row vector $d=\left(d_{1}, \ldots, d_{l}\right)$ with $M_{1} \cdot d= \pm 1$ and $M_{i} \cdot d=0$ for $i=2, \ldots, l$. Since $M_{l+1}, \ldots, M_{k}$ lie in the span of $M_{2}, \ldots, M_{l}$, it is even true that $M_{i} \cdot d=0$ for $i=2, \ldots, k$. This proves (3) if $d_{j} \neq 0$ for $j=1, \ldots, l$. Otherwise, reorder the columns of $M$ if necessary so that $d_{j}=0$ for $j=1, \ldots, l^{\prime}$ and $d_{j} \neq 0$ for $j=l^{\prime}+1, \ldots, l$ for some $l^{\prime}$. Let $M^{\prime}$ be the $k \times l^{\prime}$ matrix consisting of the first $l^{\prime}$ columns of $M$. Note that there exists an index $j_{0}$ with $d_{j_{0}} \neq 0$ and the $j_{0}$ th entry of the first row $M_{1}$ of $M$ is also nonzero.

Further suppose that this $j_{0}$ th column of $M$ is not an elementary column. Then $M^{\prime}$ satisfies the assumptions of (3) by (5) and $M^{\prime}$ has fewer nonelementary columns than $M$, and so the induction hypothesis applies to $M^{\prime}$. Thus there exist $e_{1}= \pm 1, \ldots, e_{l^{\prime}}= \pm 1$ such that the inner products of the rows of $M^{\prime}$ with $\left(e_{1}, \ldots, e_{l^{\prime}}\right)$ lie in $\{-1,0,1\}$. Taking $e_{j}=d_{j}$ for $j=l^{\prime}+1, \ldots, l$ or $e_{j}=-d_{j}$ for $j=l^{\prime}+1, \ldots, l$ gives a vector $e=\left(e_{1}, \ldots, e_{l}\right)$ with $M_{i} \cdot e \in\{-1,0,1\}$ for $i=1, \ldots, k$. This proves (3) in this case.

Now suppose that the $j_{0}$ th column of $M$ is an elementary column. Deleting this column produces a $k \times(l-1)$ matrix which by (5) satisfies the assumptions of (3). As in the previous paragraph, if the conclusion of (3) holds for this smaller matrix, then it holds for $M$. Furthermore,

this new matrix has one fewer rows than $M$ whose inner products with $(1,1,1, \ldots, 1)$ are congruent to 1 modulo 2 .

The proof of (3) will now be completed by inducting on $s$, the number of rows $M_{i}$ in $M$ with $M_{i} \cdot(1,1,1, \ldots, 1) \equiv 1 \bmod 2$. Suppose that $s=1$. Then $M_{i} \cdot(1,1,1, \ldots, 1) \equiv 0 \bmod 2$ for $i=2, \ldots, k$. It follows from (4) that the dimension of the span of $M_{2}, \ldots, M_{k}$ is $l-1$ since this is true modulo 2. Thus the argument from (6) to (7) proves (3) for the case $s=1$. Suppose that $s>1$ and that (3) holds for smaller values of $s$. If $(6)$ is true, then the argument from (6) to (7) again gives (3). If (6) is not true, then enlarge $M$ by adjoining an $(l+1)$ th column which is the elementary column with 1 in its first entry. This $k \times(l+1)$ matrix $M^{\prime}$ satisfies the assumptions of $(3)$, it has the same number of nonelementary columns as $M$ but it has a smaller value of $s$. 
Thus the induction hypothesis applies to $M^{\prime}$ giving $e^{\prime}=\left(e_{1}, \ldots, e_{l+1}\right)$ with $e_{j}= \pm 1$ for $j=1, \ldots, l+1$ such that the inner product of the $i$ th row of $M_{i}^{\prime}$ of $M^{\prime}$ with $e^{\prime}$ lies in $\{-1,0,1\}$ for $i=1, \ldots, k$. Set $e=\left(e_{1}, \ldots, e_{l}\right)$. Since $M_{1}^{\prime} \cdot e^{\prime} \equiv 0 \bmod 2$, it follows that $M_{1}^{\prime} \cdot e^{\prime}=0$, and so $M_{1} \cdot e= \pm 1$. Thus $M_{i} \cdot e$ lies in $\{-1,0,1\}$ for $i=1, \ldots, k$. This concludes the proof of (3) and the theorem.

\section{REFERENCE}

1. C. Gordon and J. Luecke, Knots are determined by their complements, J. Amer. Math. Soc. 2 (1989), 371-415.

Department of Mathematics, Eastern Michigan University, Ypsilanti, Michigan 48197 